\title{
Assessing the Dimensionality of a Set of Test ltems
}

\author{
Ronald K. Hambleton \\ University of Massachusetts at Amherst \\ Richard J. Rovmelli \\ Educational Services for the Professions
}

\begin{abstract}
This study compared four methods of determining the dimensionality of a set of test items: linear factor analysis, nonlinear factor analysis, residual analysis, and a method developed by Bejar (1980). Five artificial test datasets (for 40 items and 1,500 examinees) were generated to be consistent with the three-parameter logistic model and the assumption of either a oneor a two-dimensional latent space. Two variables were manipulated: (1) the correlation between the traits $(r=.10$ or $r=.60)$ and (2) the percent of test items measuring each trait (50\% measuring each trait, or $75 \%$ measuring the first trait and $25 \%$ measuring the second trait).

While linear factor analysis in all instances over-
\end{abstract}

\begin{abstract}
estimated the number of underlying dimensions in the data, nonlinear factor analysis with linear and quadratic terms led to correct determination of the item dimensionality in the three datasets where it was used. Both the residual analysis method and Bejar's method proved disappointing. These results suggest the need for extreme caution in using linear factor analysis, residual analysis, and Bejar's method until more investigations of these methods can confirm their adequacy. Nonlinear factor analysis appears to be the most promising of the four methods, but more experience in applying the method seems necessary before wide-scale use can be recommended.
\end{abstract}

The assumption that a set of test items is "unidimensional" is made for all of the currently popular item response models. Despite the importance of the assumption to these models, there is substantial confusion in the psychometric literature concerning the proper definition of the term "unidimensionality" and the methods for assessing its presence or absence in a set of test items (Hattie, 1984, 1985; Traub \& Wolfe, 1981). Such definitions of unidimensionality are typically abstract and non-operational. A typical example is: A set of test items is unidimensional when a single trait can explain or account for examinee test performance.

Methods for assessing the unidimensionality of a set of test items range from the commonly used but unacceptable measures of internal consistency (Green, Lissitz, \& Mulaik, 1977) to the more appropriate uses of eigenvalue plots and related statistics (Lord \& Novick, 1968; Reckase, 1979). Hattie (1984) reported that there are 87 indices in the psychometric literature for addressing the dimensionality of a set of test items. Unfortunately, many methods for assessing the unidimensionality of a set of test items are only loosely connected to the various definitions in the psychometric literature.

APPLIED PSYCHOLOGICAL MEASUREMENT

Vol. 10, No. 3, September 1986, pp. 287-302

(C) Copyright 1986 Applied Psychological Measurement Inc.

$0146-6216 / 86 / 030287-16 \$ 2.05$ 
This investigation of several methods for assessing the unidimensionality of a set of test items was prompted by a practical problem which arose in connection with the recertification exam produced by the American Board of Family Practice (ABFP). Candidates are required to take a core exam plus three additional subtests of their choice, selected from a set of six available subtests. These six subtests vary somewhat in their difficulity. Score reporting and subsequent comparisons among candidates must be carried out on the combined exam (core plus subtests); because the candidates do not, in general, take the same three subtests (there are 20 possible combinations), the subtests are equated to the common scale defined by the core items. The degree of success of the equating of candidate scores depends upon the choice of item response model (the three-parameter model is chosen to increase the likelihood of a satisfactory model fit to the data) and the extent to which the core items and subtest items measure a common trait, that is, a unidimensional trait. Thus, two questions arose: "What is meant by the expression "unidimensionality of a set of test items"?" and "How should the assumption of unidimensionality be assessed to be consistent with the definition?"

Despite the confusion in the literature on these two questions, there are contributions by McDonald $(1980,1982)$ and Hattie (1981) which proved to be helpful, and these contributions influenced the general direction of the present research. McDonald and Hattie arrived at the conclusion that the principle of local independence should be the basis for a proper definition for the assumption of unidimensionality. McDonald defined a set of test items as unidimensional if, for examinees with the same ability, the covariation between items in the set is zero. Since the relations between items are typically nonlinear, he recommended the use of nonlinear factor analysis to study these relations. Also, after fitting a single nonlinear factor model to the item set, he recommended that residual covariances be calculated and used to assess the plausibility of the unidimensionality assumption. McDonald argued that the dimensionality of a set of test items should be determined by the number of factors or traits needed for describing examinees, so that the principle of local independence is satisfied.

This research focused on three promising methods for addressing the unidimensionality of a set of test items: (1) nonlinear factor analysis (NLFA), because of McDonald's recommendation; (2) residual analysis; and (3) the Bejar (1980) analysis method. The first method appeared promising because NLFA does not require the implausible assumption of linear relationships among the variables, and between the variables and the underlying traits. In fact, one of the fundamental assumptions of item response theory (IRT) is that these relationships are nonlinear (Lord, 1980). The second method is an assessment of the overall fit of a unidimensional model to a dataset through the analysis of residuals. When the fit is adequate it would seem that the assumption of a unidimensional model is also plausible (e.g., Rentz \& Rentz, 1979). Of course, when the fit is poor, specific reasons for the misfit may be unknown. For example, the assumption of unidimensionality may not be violated by the dataset even though some other assumption of the model is violated. The Bejar (1980) method appeared useful for assessing item dimensionality because it does not involve questionable linearity assumptions about the test data. Also, the method provides a straightforward check on one of the expected outcomes of a unidimensional set of test data: The subset of items from a test in which an item is calibrated is irrelevant. A fourth assessment method, linear factor analysis, was also studied to provide a basis for comparing the merits of the methods. The four methods were applied to five datasets. The datasets were artificial and were generated to reflect oneand two-dimensional datasets.

\section{Methods for Assessing ltem Dimensionality}

\section{Linear Factor Analysis}

Linear factor analysis (LFA) is probably the most commonly used method for studying item dimensionality. Using (1) the matrix of phi or tetrachoric correlations to summarize the linear relationships 
between pairs of items in a test ${ }^{1}$, and (2) communality estimates (often, squared multiple correlations) in the diagonal entries of the correlation matrix, eigenvalues are extracted from the correlation matrix and plotted (from largest to smallest). The number of "significant" factors is determined by looking for the "elbow" in the plot. The number of eigenvalues to the left of the elbow is normally taken to be the number of significant factors underlying test performance. The method fails in those instances where an elbow cannot be found.

A second procedure for determining the number of factors is the application of an identical LFA to a dataset consisting of the same number of items and examinees, but with random normal deviates substituted for the actual data. This "parallel analysis" procedure appears to have been first suggested by Horn (1965) and was later studied by Linn (1968), Humphreys and Ilgen (1969), and Humphreys and Montanelli (1975). For the purpose of computing phi and tetrachoric correlations, the data are dichotomized at $z=0$; normal deviates above 0 are coded as "correct" and below 0 as "incorrect". ${ }^{2}$ Since all of the data are randomly generated, the value of the largest eigenvalue can be used as a cutoff score with the eigenvalues obtained from the actual data to determine the number of significant factors. Thus, if the largest eigenvalue obtained from the random data is 1.5 , all eigenvalues over 1.5 in the analysis of the real data are considered to be associated with significant factors.

\section{Nonlinear Factor Analysis}

McDonald (1967) sought to improve upon LFA by developing nonlinear factor analysis (NLFA). In NLFA, nonlinear relationships between the variables and the traits or factors measured by the variables are assumed. The application of NLFA to the study of item dimensionality seems especially desirable in an IRT context because one of the principal assumptions-the mathematical form of the item characteristic curves (ICCS) - specifies a particular nonlinear relationship between item performance and ability. One version of NLFA takes the form

$y_{i}=a_{i 0}+\sum_{\ell=1}^{t} \sum_{p=1}^{s} a_{i \ell} \theta_{\ell}^{p} \quad(i=1,2, \ldots, n) \quad$,

where $y_{i}$ is an examinee's score on item $i$,

$t$ is the number of traits necessary to account for examinee test performance,

$s$ is the degree of the polynomial used to fit the model with each factor, and

$a_{i t_{p}}$ is the factor loading of the $i$ th item on the $\mathrm{th}$ trait for the $p$ th degree element in the polynomial.

For example, with a one-factor model $(t=1)$ with polynomial terms to the third power $(s=3)$, the model has the following form:

$y_{i}=a_{i 0}+a_{i 11} \theta_{1}+a_{i 12} \theta_{1}^{2}+a_{i 13} \theta_{1}^{3} \quad(i=1,2, \ldots, n)$.

The two-factor model $(t=2)$ with polynomial terms to the third power $(s=3)$ has the form

$y_{i}=a_{i 0}+a_{i 11} \theta_{1}+a_{i 12} \theta_{1}^{2}+a_{i 13} \theta_{1}^{3}+a_{i 21} \theta_{2}+a_{i 22} \theta_{2}^{2}+a_{i 23} \theta_{2}^{3} \quad(i=1,2, \ldots, n)$.

The one- and two-factor models above with linear, with linear and quadratic, and with linear, quadratic, and cubic terms were fitted to the various datasets in the study.

\footnotetext{
${ }^{1}$ Usually the choice of phi correlations or tetrachoric correlations is not a major consideration in conducting factor analysis studies. But McDonald (personal communication, June 24, 1983) notes that as Lord originally showed, if the normal ogive model fits the data, and if ability scores are normally distributed, the matrix of tetrachoric correlations should fit a Spearman model. This is therefore an approximate method for fitting the normal ogive model.

${ }^{2}$ In several pilot runs, the $z$ scores were dichotomized to simulate the actual difficulty levels of the test items. However, the effect on the results was minimal.
} 


\section{Residual Analysis}

The method for addressing the unidimensionality of a set of test items through a residual analysis involves fitting a unidimensional IRT model of interest to the test data, using the model parameter estimates to predict the item performance data, and then summarizing the discrepancies or residuals (e.g., Hambleton \& Swaminathan, 1985). Specifically, $\theta$ categories are chosen to divide the $\theta$ scale into equal intervals. Examinees are assigned to categories based upon their $\theta$ estimates. For examinees in each $\theta$ category on each item, a comparison is made between actual performance (proportion correct) and the predicted proportion-correct level from the corresponding ICC. In this study the proportion-correct estimate was obtained at the midpoint of each $\theta$ category. (A slightly better estimate is the average of the probabilities for a correct answer associated with the $\theta$ estimates for examinees in the category.) The difference between the actual and predicted proportion-correct score (called a residual or a raw residual score) in each $\theta$ category and for each item can also be divided by the corresponding standard error of the proportioncorrect estimate to obtain a standardized residual (SR). When the chosen model fits the dataset, these SRS might be expected to be small and randomly distributed about 0.0 . It is common within the framework of regression theory to assume that the distribution of SRs is normal. Of course, the distribution of residuals would only be (at best) approximately normal because of non-normall distributions of the SRS when the ICCs approach values of 0 or 1 . The rationale for the appropriateness of residuals as a check on item unidimensionality is that when a unidimensional model fits a dataset, all of the model assumptions must be met to a reasonable degree.

\section{Bejar Analysis}

Bejar (1980) argued that if the set of items in a test is unidimensional, then the grouping of test items for the purpose of item calibration will be irrelevant. Parameter estimates for items calibrated with different subsets of items, aside from sampling errors, should be identical. Bejar's method (with minor modifications) can be implemented in four steps:

1. Identify a subset of items which appears to be measuring a trait different from the trait measured by the total test.

2. Conduct a three-parameter model analysis of only the items in the subtest.

3. Repeat the three-parameter model analysis using the total set of items.

4. Compare the two sets of $b$-value estimates for items in the subtest. (Because $b$ values are estimated with smaller sampling errors than the $a$ values, $b$ values are more useful for studying the relationships between item parameter estimates obtained in two samples.) Bejar has provided a simple statistical test that can be used to compare the parameter estimates. Alternatively, the pairs of $b$ values can be plotted to determine the extent to which the two sets are linearly related.

The pairs of parameter estimates for items in the subtest and test, respectively, should be linearly related unless the subset of items measures a trait or traits which are not common to the trait or traits measured in the total test.

\section{Method}

\section{Criteria for Assessing the Usefulness of the Methods}

Linear factor analysis. One way to evaluate LFA as a measure of item dimensionality is to compare the number of factors retained in a solution to the dimensionality of the latent space in the artificial data. Two ways for determining the number of factors were used: (1) the elbow in the plot of eigenvalues; 
(2) eigenvalues greater than the largest eigenvalues obtained with the random data. Also, to facilitate a comparison between LFA and NLFA, four additional criteria were used. After fiting one, two, three, four, and five factors to the reduced correlation matrix, the matrix of residuals was calculated. The off-diagonal elements of the matrix were summarized by (1) the average residual, (2) the standard deviation of the residuals, (3) the average of the absolute-valued residuals, and (4) the standard deviation of the absolutevalued residuals. When the chosen model fits the dataset well, the true values of these off-diagonal elements are (near) zero. In practice, because of errors of measurement and of sampling, the residuals should be small, and evenly distributed around 0.0 .

Nonlinear factor analysis. The four statistics described above were calculated after fitting one-and two-factor models with linear, quadratic, and cubic terms to the inter-item correlation matrices for four of the five datasets.

Residual analysis. For each arificial dataset there were 40 items and $12 \theta$ categories. In total, 480 residuals were produced. Since interest was on the size and not the direction of the discrepancies, absolutevalued residuals (both raw and standardized) were substituted for residuals and standardized residuals. Criterion measures chosen were (1) average absolute-valued residual, (2) average absolute-valued standardized residual, and (3) the distribution of absolute-valued standardized residuals. With respect to the second of these criteria, when a model fits the data and the SRS are normally distributed, then the average absolute-valued SR should be close to .799 . (The value of .799 can be obtained by calculating the average of absolute values of normal deviates.) Because a study of absolute-valued residuals is more informative when several models have been fit to the same test data, residual analyses were carried out with the one-, two-, and three-parameter unidimensional logistic models. A computer program described by Hambleton (1982) was. used to conduct the residual analyses.

A comparable analysis of residuals with NLFA would have been useful, but with the polynomial models the expected probabilities are not defined on the interval $[0,1]$ across the total $\theta$ score range, and therefore an analysis of residuals would have no value.

Bejar analysis. The results from this analysis were summarized by a correlation coefficient between $b$ values for a subset of test items: items calibrated in a subtest and again in the total test. The original plan was to produce the plot of item $b$ values obtained in the subtest and total test for each of the five datasets, but the correlations were very high in all but one analysis, and therefore the plots did not seem necessary.

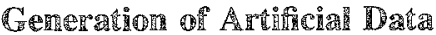

Using item parameters which were generated according to the specifications described below, for each examinee $j$, and given $\theta_{j}$, a vector of probabilities associated with answering the test items correctly $\left(P_{1}, P_{2}, \ldots, P_{n}\right)$ was obtained from the expression

$P_{i}\left(\theta_{j}\right)=c_{i}+\left(1-c_{i}\right)\left\{1+\exp \left[-D a_{i}\left(\theta_{j}-b_{i}\right)\right]\right\}^{-1} \quad(i=1,2, \ldots, n) \quad$.

Using a random number generator to produce numbers uniformly distributed on the interval $[0,1]$, the probabilities were converted to either os or $1 \mathrm{~s}$ to reflect examinee item scores. This was done by assigning $a$ " 1 " to an examinee for item $i$ when the random number selected was below $P_{i}$ (which occurs $P_{i}$ of the time), and a " 0 " otherwise.

The simulation of two-dimensional data was a substantially more difficult problem. First, there are several possible multidimensional models from which to select. Second, there are no guidelines in the IRT literature for choosing reasonable item parameter values for multidimensional models.

Sympson (1978) offered one model which took the form

$P_{i}\left(\theta_{j 1}, \theta_{j 2}, \ldots, \theta_{j k}\right)=c_{i}+\left(1-c_{i}\right) \prod_{k=1}^{K}\left\{1+\exp \left[-D a_{i k}\left(\theta_{j k}-b_{i k}\right)\right]\right\}^{-1}$. 
The problem encountered in applying this model was that the probabilities, even for the two-dimensional model $(k=2)$, quickly converged to the value $c_{i}$; consequently, there was little variation in item performance for examinees, and little variation in test scores among examinees.

A similar problem was encountered in applying a model used by Christoffersson (1975) and Hattie (1981). In Hattie's formulation,

$P_{i}\left(\theta_{j 1}, \theta_{j 2}, \ldots, \theta_{j k}\right)=c+(1-c)\left[1+\exp \left(-D \sum_{k=1}^{K} a_{i k} \theta_{j k}-b_{i}\right)\right]^{-1}$,

where

$c$ is a constant value over all items,

$b_{i}$ is the item difficulty,

$a_{i k}(k=1,2, \ldots, K)$ are the item discriminations on the $K$ underlying traits, and

$\theta_{j k}(k=1,2, \ldots, K)$ are the trait scores for examinee $j$ on the $K$ traits.

In preliminary simulations it was observed that the probabilities for small changes in $\theta$ quickly approached values of $c$ and 1 . Perhaps the main problem encountered concerned the choice of item discriminations, which were taken to be values often observed with one-dimensional models.

A simpler model than the models proposed by Sympson and Hattie was chosen. For this simpler model (a special case of the Christoffersson-Hattie model with independent item clusters), guidelines for selecting item parameters were readily available. Specifically, item parameter values were assigned to all test items in the same way that the assignments were made with the one-dimensional model described below. Then, examinees were assigned two trait scores (with the specified correlation).

The simulation of two traits used a formula developed by Hoffman (1959). First, two uncorrelated normally distributed random variables, $X_{j}$ and $Z_{j}$ (mean $=0, \mathrm{SD}=1$ ), were generated with the aid of a random number generator. Then the variable $Y_{j}$ was obtained from the expression

$Y_{j}=X_{j}+(k / r) Z_{j}$

where $k=\left(1-r^{2}\right)^{1 / 2}$ and $r$ is the desired correlation between variables $X_{j}$ and $Y_{j} . X_{j}$ and $Y_{j}$ were used in the simulations as the two trait scores for an examinee. Pairs of trait scores with the desired correlation were generated for the 1,500 examinees. Finally, item probabilities and item scores were generated for examinees. For the first 20 items (or, for Datasets 3 and 5, 30 items), the first set of trait scores was used in generating item probabilities; for the remaining items, the second set of trait scores for examinees was used.

In summary, artificial data generated from a one-dimensional model were used, in order to determine whether or not the four methods could identify unidimensional data. Additionally, two-dimensional data were used to represent situations where items could be organized into two clusters measuring different traits. Two variables were manipulated: the correlation between the traits and the percentage of total items in each cluster.

\section{Test Dakat}

The five artificial test datasets were generated to be consistent with the assumption of either a oneor a two-dimensional latent space. Each test consisted of 40 test items. The item performance for 1,500 examinees was simulated with the three-parameter logistic model. These numbers were assumed to be large enough to avoid parameter estimation problems when using LOGIST. In Dataset 1 , the latent space was assumed to be one-dimensional. In Datasets 2 through 5, the latent space was chosen to be twodimensional. Among these four datasets, the only difference was that in Datasets 2 and 3 the correlation between the two latent traits was. 10 , whereas in Datasets 4 and 5 the correlation between the two traits was .60. In addition, items were assumed to measure one trait or the other. In Datasets 2 and 4, the first 20 items measured Trait 1 and the second 20 items measured Trait 2. In Datasets 3 and 5, the first 30 
items measured Trait 1 and the remaining 10 items measured Trait 2. Table 1 summarizes the pertinent information.

Parameter values were assigned to items on each trait in the following way:

$b$ parameters were drawn at random from a uniform distribution on the interval $[-2.0$ to +2.0$]$;

a parameters were drawn at random from a uniform distribution on the interval $[.40,2.00]$;

$c$ parameters were set to a value of .25 .

The choice of item parameters reflected values often found in practice (Hambleton \& Swaminathan, 1985).

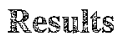

\section{One-Dimensional Data}

Linear factor analyses. The eigenvalues for the random data and the one-dimensional data are reported in Table 2. The plots of eigenvalues for the one-dimensional data, using phi correlations (or tetrachoric correlations) to measure the relationships between pairs of items, suggest that two significant factors are present. The analysis of the tetrachoric correlations was more revealing than the phi correlations in the sense that more of the variance was associated with the first factor, which would be expected when the data are unidimensional. Whether the criterion for the number of significant factors is determined from the elbows in the plots or from the largest eigenvalue from the matrix of correlations of normal random deviates, two factors would be retained.

Nonlinear factor analyses. The results of fitting from one to five linear factors, and one and two factors with linear, linear and quadratic, and linear, quadratic, and cubic terms to the one-dimensional dataset are reported in Table 3 . The first two criteria, $\bar{r}_{i j}$ and $s\left(r_{i j}\right)$, show simply that the mean off-diagonal clements, after fitting one or more factors, are centered close to .00 (as compared to .127 in the original correlation matrix) and that the standard deviation of the distribution of the residuals approaches zero as the number of factors is increased. From the statistics in the third and fourth columns of Table 3, it is clear that a NLFA with one factor with linear and quadratic terms fits the data better than the two-factor solution provided by LFA. In fact, even three linear factors did not produce as accurate a tit to the data.

Residual analyses. The residual analyses for the one-dimensional data with the three logistic models are reported in Table 4. Not surprisingly, since the data were generated to fit the three-parameter model, this model provided the best fit to the data. More importantly, the distribution of SRs was approximately

Table 1

Surnary of parameter Values

\begin{tabular}{|c|c|c|c|c|}
\hline \multirow[b]{2}{*}{ Dataset } & \multirow[b]{2}{*}{ Trait(s) } & \multirow[b]{2}{*}{$r\left(\theta_{1}, \theta_{2}\right)$} & \multicolumn{2}{|c|}{ Number of Items } \\
\hline & & & First Trait & Second Trait \\
\hline 1 & $\mathbb{1}$ & $\infty$ & 40 & 0 \\
\hline 2 & 2 & .10 & 20 & 20 \\
\hline 3 & 2 & .10 & 30 & 10 \\
\hline 4 & 2 & .60 & 20 & 20 \\
\hline 5 & 2 & .60 & 30 & 10 \\
\hline
\end{tabular}

Downloaded from the Digital Conservancy at the University of Minnesota, http://purl.umn.edu/93227. May be reproduced with no cost by students and faculty for academic use. Non-academic reproduction requires payment of royalties through the Copyright Clearance Center, http://www.copyright.com/ 


\begin{tabular}{|c|c|c|c|c|c|c|}
\hline \multirow[b]{3}{*}{ Factor } & \multirow{2}{*}{\multicolumn{2}{|c|}{$\begin{array}{l}\text { Random Data } 1 \\
\text { Tetrachoric }\end{array}$}} & \multicolumn{4}{|c|}{ One-Dimensional Data } \\
\hline & & & \multicolumn{2}{|c|}{ Phi } & \multicolumn{2}{|c|}{ Tetrachoric } \\
\hline & $\lambda$ & $\%$ & $\lambda$ & $\%$ & $\lambda$ & $\%$ \\
\hline 1 & 1.48 & 3.7 & 8.86 & 22.2 & 15.00 & 37.5 \\
\hline 2 & 1.44 & 3.6 & 2.09 & 5.2 & 2.21 & 5.5 \\
\hline 3 & 1.37 & 3.4 & 1.11 & 2.8 & 1.15 & 2.9 \\
\hline 4 & 1.34 & 3.3 & 1.05 & 2.6 & 1.10 & 2.7 \\
\hline 5 & 1.32 & 3.3 & 1.03 & 2.6 & 1.08 & 2.7 \\
\hline 6 & 1.30 & & 1.02 & & 1.02 & \\
\hline 7 & 1.28 & & .98 & & .96 & \\
\hline 8 & 1.25 & & .96 & & .95 & \\
\hline 9 & 1.22 & & .95 & & .92 & \\
\hline 10 & 1.21 & & .93 & & .90 & \\
\hline 11 & 1.19 & & .93 & & .88 & \\
\hline 12 & 1.18 & & .93 & & .84 & \\
\hline 13 & 1.15 & & .91 & & .80 & \\
\hline 14 & 1.13 & & .89 & & .77 & \\
\hline 15 & 1.10 & & .86 & & .74 & \\
\hline
\end{tabular}

1 Squared multiple correlations used as communality estimates.

normal and the mean absolute-valued SR was close to .799 . With the one-dimensional data and when the particular IRT model closely fits the data, the SRS appear to have the desired distribution. The slight predicted bias in this distribution is also apparent.

Bejar analyses. Because all of the test items were generated to fit a one-dimensional model, there was no reason to suspect that a second trait was necessary to account for the inter-item correlations. As a rather simple check on the method, the last 20 items were presumed to measure a second trait. The $b$ values for the last 20 items (calibrated both in the subtest composed of the last 20 items and the total test) are reported in Table 5. The correlation between the $b$ values was in excess of .99. Clearly, the assumption of unidimensionality could not be rejected on the basis of the available evidence, nor should the assumption be rejected for this dataset.

\section{Two-Dimensional $\mathrm{Data}$}

Linear factor analyses. If the largest eigenvalue of the random data $\left(\lambda_{1}=1.48\right)$ is used as the criterion for determining the number of factors, for all 4 two-dimensional datasets three significant factors emerged (see Table 3). If the elbow of each eigenvalue plot is used, possibly two significant factors 
R. K. HAMBLETON AND R. J. ROVINELLI ASSESSING TEST ITEM DIMENSIONALITY

Table 3

Residual Matrices After Fitting Linear and Non-Linear Factor Analys is Models

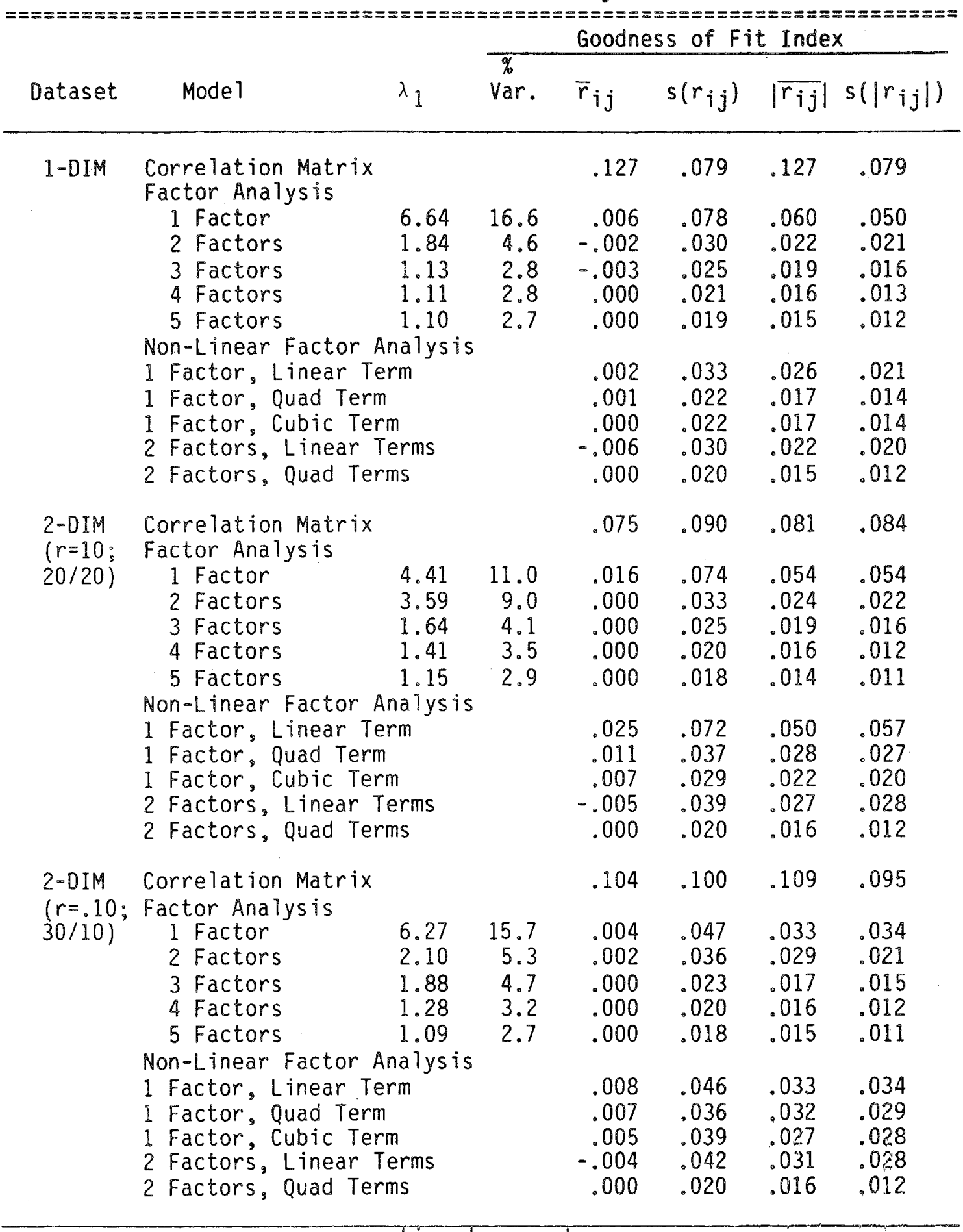




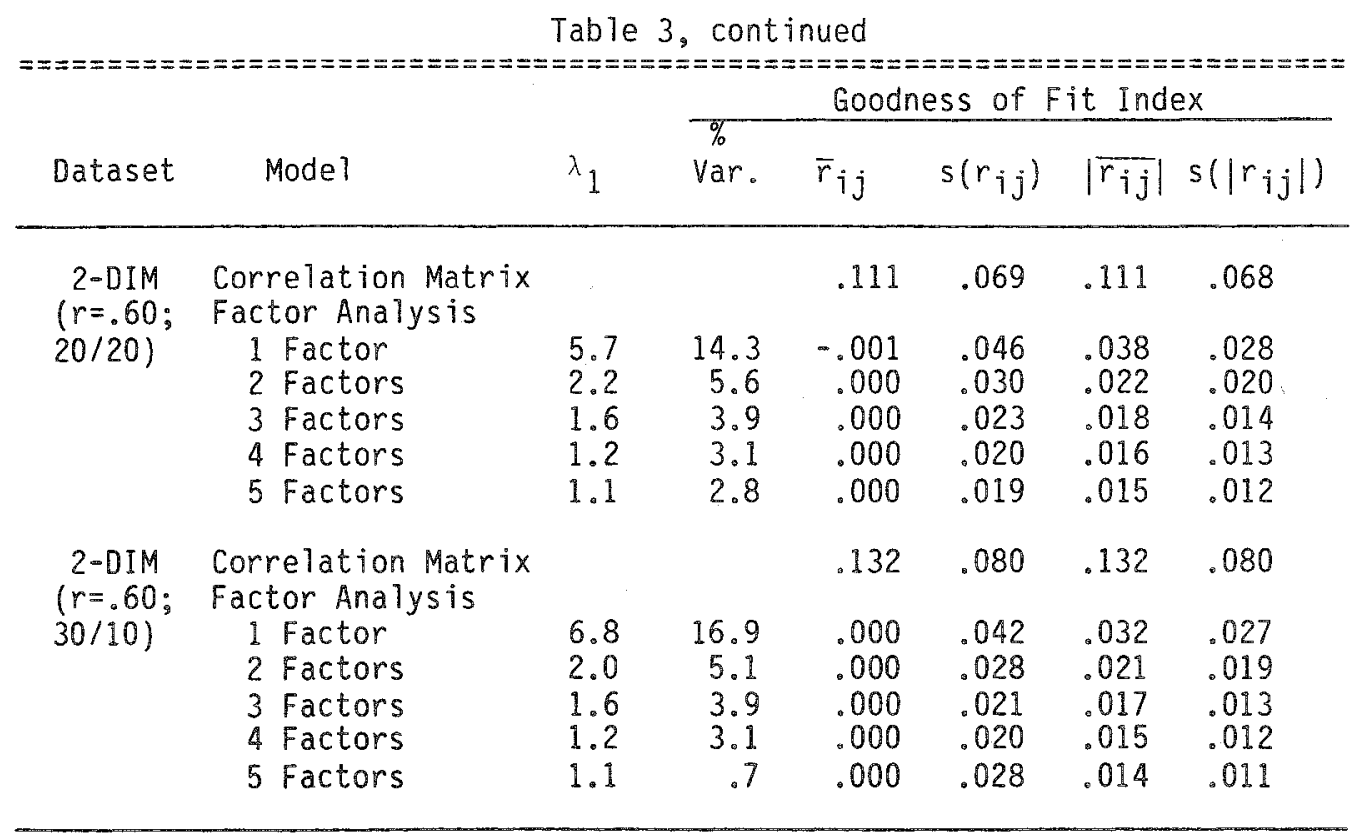

might emerge for the two-dimensional data $(r=.10 ; 20 / 20)$, but choosing three or four factors is not totally unreasonable. With the other two-dimensional datasets, the elbow also revealed at least three significant factors. Again, the linear factor analysis method resulted in more factors than the underlying dimensionality of the data. However, LFA did reveal the dominance of one factor over the other. With the two-dimensional data $(r=.10 ; 20 / 20)$, the first two factors accounted for roughly the same amount of variance. With the two-dimensional data $(r=.10 ; 30 / 10)$, the ratio of variance accounted for by the first two factors (15.7/5.3 or roughly 3/1) was proportional to the number of items measuring each factor $(30 / 10$ or $3 / 1)$. For the final two datasets a second-order factor appeared to be emerging.

Nonlinear factor analyses. Again, Table 3 shows that NLFA provided promising results. With the two-dimensional data $(r=.10 ; 20 / 20)$ and the two-dimensional data $(r=.10 ; 30 / 10)$, the mean and standard deviation of absolute-valued residuals associated with a two-factor model with quadratic terms were smaller than the corresponding residuals obtained from a three-factor solution using LFA. Thus, if the three-factor solution with LFA is acceptable, then the two-factor solution from NLFA would be as well. The two-factor model with cubic terms was not obtained because of the high costs associated with rumning the computer program and the acceptability of the two-factor solution with quadratic terms.

Residual analyses. Table 4 provides a summary of the absolute-valued residuals and standardized residuals obtained from fitting logistic models to the 4 two-dimensional datasets. Several findings are evident:

1. The one-parameter model did not fit any of the datasets. However, rather than suggesting multidimensionality in the data, the likely explanation, in view of the results of fitting the one-parameter model to the one-dimensional data (and point two below), is that the misfit is due to the failure of the model to account for variations in item discriminations and the guessing behavior of low-ability examinees. 


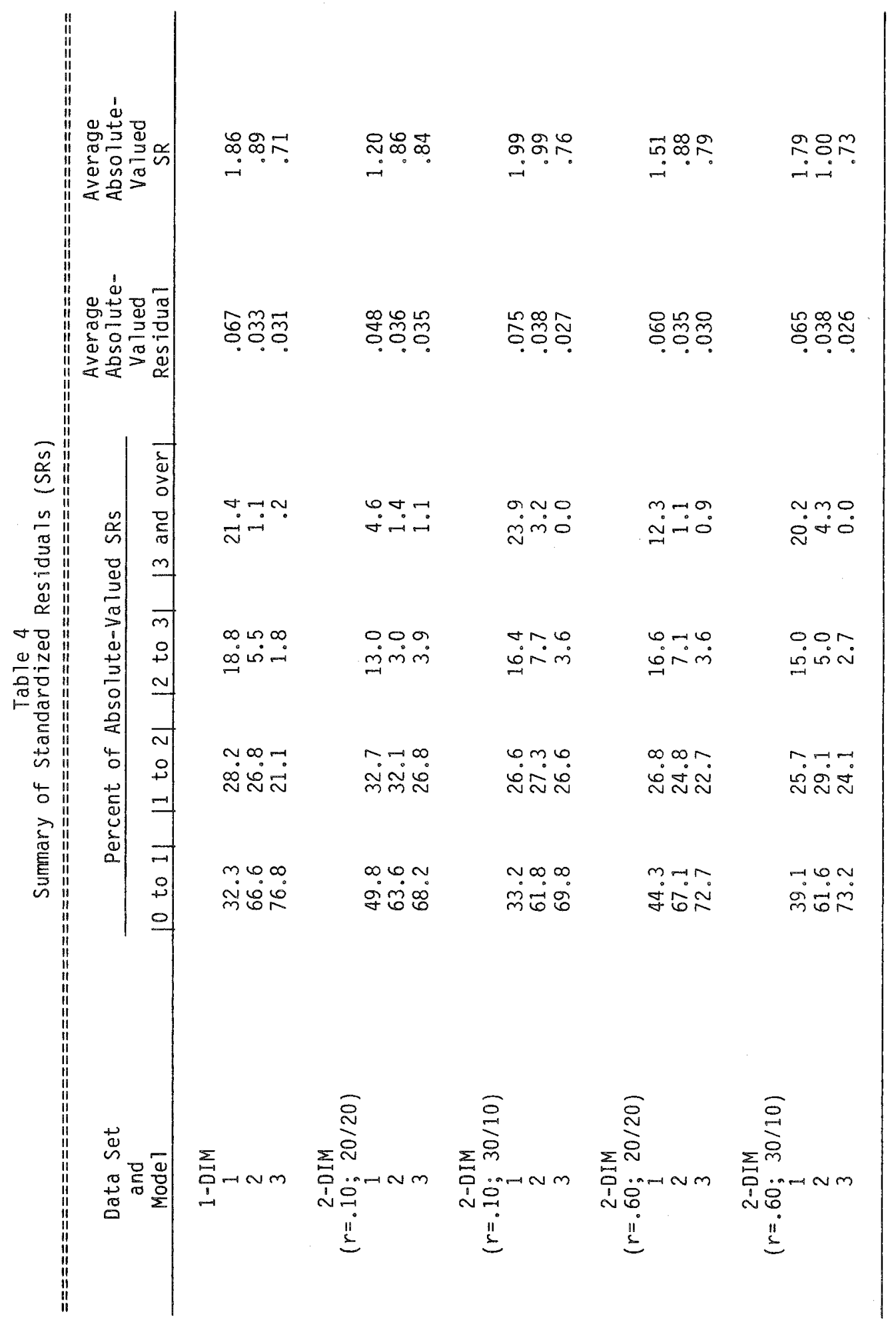
requires payment of royalties through the Copyright Clearance Center, http://www.copyright.com/ 


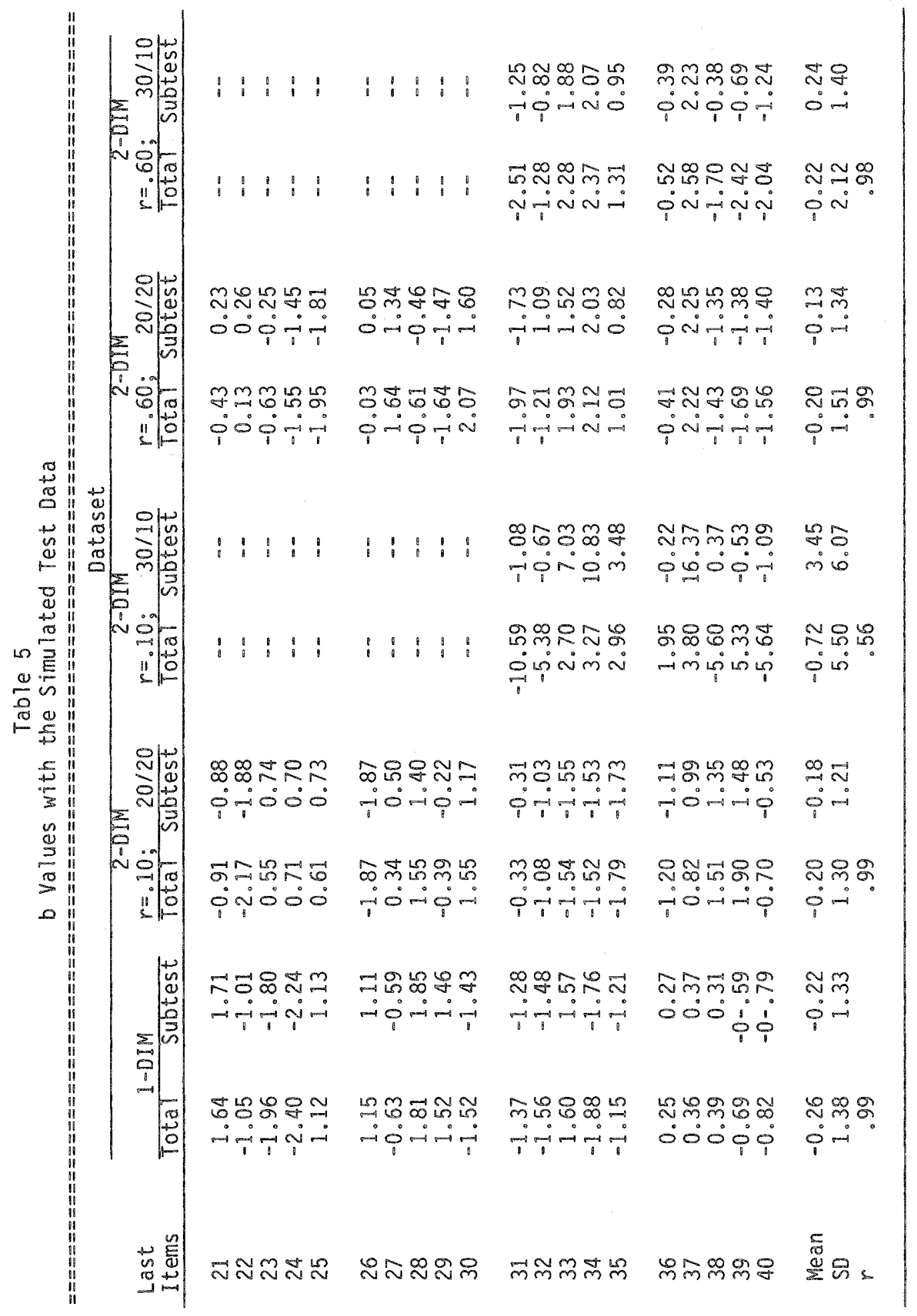


2. A comparison of the SRs from the two- and three-parameter models showed substantially smaller SRS than those obtained with the one-parameter model, and showed the three-parameter model to fit the datasets slightly better than the two-parameter model. In fact, on the basis of examination of the SRS for the two- and three-parameter models, and assuming the validity of the residual analysis method, a researcher would accept the hypothesis that the test items in each dataset were unidimensional.

3. There was also evidence that when the traits were correlated $(r=.60)$, the overall fits were better than when the traits were not $(r=.10)$. The average absolute-valued standardized residual dropped from .84 and .76 (in Datasets 2 and 3, with $r=.10$ ) to .79 and .73 (in Datasets 4 and 5, with $r$ $=.60$ ), respectively.

How could the three-parameter model fit the 4 two-dimensional datasets? The failure to identify multidimensionality in Datasets 4 and 5 was surprising, but in view of the moderately high correlation between the two traits, the results were not totally unexpected although larger SRs had been predicted. Apparently LOGIST simply proceeds to estimate the second-order factor which incorporates the two related factors. Why multidimensionality could not be detected in Datasets 2 and 3 is not completely clear. It appears that LOGIST estimates an average ability of the two unrelated traits and also attaches low $a$ values to all of the test items. In doing so, a reasonable fit between the model and each dataset can be achieved. When there is an imbalance in the test data (i.e., 30/10), LOGIST assigns high $a$ values to items measuring the "dominant trait" and relatively low values to the remaining items. In this way, a one-dimensional model can fit the data. With a more even split (i.e., 20/20), the values assigned to the $a$ values are relatively low. In any case, because of the way LOGIST handles multidimensionality in the test data, residual analyses cannot identify it when it is present.

Bejar analyses. The results of the Bejar analyses on the 4 two-dimensional datasets are reported in Table 5. For the purposes of these analyses, the last 20 items in Datasets 2 and 4 and the last 10 items in Datasets 3 and 5 constituted the subtests. These, of course, were the test items in the datasets that measured the second traits. The following observations were noted:

1 , With $r=.10$, and a split of $20 / 20$, the test items had comparable $b$ values.

2. With $r=.10$, and a split of $30 / 10$, the $b$ values were substantially different and appeared to be poorly estimated. This analysis would lead to a rejection of the unidimensionality assumption.

3. When $r=.60$, and for the two splits $20 / 20$ and $30 / 10$, the Bejar analyses suggested that the assumption of unidimensionality could not be rejected.

In only one of the four analyses was the Bejar method sensitive to the multidimensionality in the data. This result also was surprising because the method appeared to have been successful in at least one other study (Bejar, 1980).

\section{Real Data}

Though the results are not reported here, the four methods for assessing item dimensionality were also applied to the 80-item section of the 1982 ABFP recertification exam. The four methods provided different answers to the question of unidimensionality. Had the simulation studies described earlier not been done, the results from the residual analyses or the Bejar analyses would have been used to support the assumption of unidimensionality. The LFA of the data suggested that 4 to 8 significant factors would need to be retained for a satisfactory accounting of the data. The NLFA also appears to indicate that more than one factor may be needed. If, for example, the ratio of the average absolute residual (after fitting a one-factor model with a cubic term) to the average correlation in the initial matrix is used as a criterion, the ratio is $.018 / .042$ or .438 , whereas with clearly one-dimensional data the ratio was $.017 / .127$ or .134 . It would seem that more than one factor is needed to obtain a satisfactory solution. 
The four methods provided contradictory information about the item dimensionality in these data. Based upon the results from the simulations, it would seem that the most likely conclusion is that more than one dimension is operating.

\section{Discussion}

On the basis of a single simulation study with limited scope, generalizability of the findings is obviously limited. But several findings of the study do appear to suggest some directions for future work. First, the linear factor analysis model in all instances overestimated the number of underlying dimensions in the data. Of course, this result, along with the result that the tetrachoric correlations are more useful than phi correlations in addressing item dimensionality, is well-known. Second, nonlinear factor analysis with linear and quadratic terms led to the correct determination of the item dimensionality in the three datasets where it was used. A problem that emerged in the use of NLFA was the appropriate number of factors and polynomial terms to retain in a solution. In this study, this decision was determined by comparing the size of the residuals to those obtained from a satisfactory linear factor analysis. When working with real data, another criterion will be needed to determine the adequacy of model fit. McDonald (personal communication) has indicated that the standard error of a binary covariance gives a good indication of the expected sizes of the residual covariances.

Both the residual analysis method and Bejar's method provided disappointing results. The residual analysis method using the one-parameter model is certainly of limited value in addressing item dimensionality because large residuals may be due to the violations of several model assumptions, including unidimensionality. This problem can be reduced somewhat by fitting a three-parameter logistic model to the dataset. But even in the two-dimensional case where the traits were nearly orthogonal, the residual analysis method with the three-parameter model could not detect the violation in the unidimensionality assumption. It appears that the three-parameter model can accommodate multidimensionality by assigning low a values to these "deviant" items. Good fit is achieved, but in doing so, the deviant items are essentially removed from the test because those items contribute little to either $\theta$ estimation or the test information function.

Likewise, the Bejar method was unable to detect the two underlying traits, except when the correlation between the traits was low and a disproportionate number of the test items measured one of the traits. A highly plausible explanation for the failure of the Bejar method with the datasets is as follows: While it is certainly true that in Datasets 2 and 3 the traits have a low correlation $(r=.10)$, there was a very high correlation between the trait scores used in calibrating test items in the subtest and the total test. In fact, if $\theta_{1}$ is assumed to measure the first trait and $\theta_{2}$ the second trait, then the trait measured by the combined set of test items might be closely approximated by $w_{1} \theta_{1}+w_{2} \theta_{2}$, where $w_{1}$ and $w_{2}$ represent the proportion of items in the test measuring Traits 1 and 2, respectively. When the subtest of items is calibrated, $\theta_{2}$ is operating; whereas when the subset of items is calibrated in the total test, $w_{1} \theta_{1}+w_{2} \theta_{2}$, and not $\theta_{1}$, is the underlying trait being measured. If scores on $\theta_{1}$ and $\theta_{2}$ are standardized (as they were in the simulations), and if $w_{1}=w_{2}=.50$, the correlation between the trait measured by the subtest (denoted $\theta_{2}$ ) and the total test (approximated by $.5 \theta_{1}+.5 \theta_{2}$ ) is at least .70 . (And when the correlation between the traits is .60 , the expected correlation between $\theta_{2}$ and $.5 \theta_{1}+.5 \theta_{2}$ is over .88.) Perhaps then it is not so surprising that the test items had highly similar difficulty estimates when calibrated in the subtest and the total test. In the only application where the Bejar method indicated a violation of the unidimensionality assumption, the correlation between $\theta_{2}$ and $w_{1} \theta_{1}+w_{2} \theta_{2}$ was relatively low because $w_{1}=.75$ and $w_{2}=.25$. Why was a similar result not obtained in Dataset 5 ? The same trend was not observed in Dataset 5 because although the weighting of test items on the two traits was disproportionate ( 3 to 1 ), the traits were moderately correlated $(r=.60)$. 
The problem identified in this study with the Bejar method can be resolved by plotting $\theta$ estimates for examinees obtained in two independent samples - one from the subtest and the other from the remaining test items. While the $b$-value plots, in theory, are preferable because of their higher precision, there is not an obvious way to remove the high overlap in the $\theta$ estimates, apart from choosing relatively short subtests in relation to the total test. But this solution also has problems, because only relatively unstable $\theta$ estimates will be available for item calibration.

Despite the limited scope of the present investigation, the results do suggest the need for extreme caution in using linear factor analysis, residual analysis, or Bejar's method to address questions about item unidimensionality. Clearly, more investigations of these methods showing some positive results are needed before they can be strongly recommended for use by practitioners. On the other hand, while nonlinear factor analysis produced the most promising results in this study, an accepted criterion for determining the minimum number of factors to retain in a nonlinear factor solution is not available, nor is an easy-to-use nonlinear factor analysis program available. More research along these lines must be carried out before NLFA can be recommended.

\section{Reererences}

Bejar, I. I. (1980). A procedure for investigating the unidimensionality of achievement tests based on item parameter estimates. Journal of Educational Measurement, 17, 283-296.

Christoffersson, A. (1975). Factor analysis of dichotomized variables. Psychometrika, 40, 5-22.

Green, S. B., Lissitz, R. W., \& Mulaik, S. A. (1977). Limitations of coefficient alpha as an index of test unidimensionality. Educational and Psychological Measurement, 37, 827-838.

Hambleton, R. K. (1982). Applications of item response models to NAEP mathematics exercise results (Final Report, ECs Contract No. 02-81-20319). Denver CO: Educational Commission of the States.

Hambleton, R. K., \& Swaminathan, H. (1985). Item response theory: Principles and applications. Boston: Kluwer-Nijhoff.

Hattie, J. A. (1981). Decision criteria for determining unidimensionality. Unpublished doctoral dissertation, University of Toronto.

Hattie, J. A. (1984). An empirical study of various indices for determining unidimensionality. Multivariate Behavioral Research, 19, 49-78.

Hattie, J. A. (1985). Methodology review: Assessing unidimensionality of tests and items. Applied Psychological Measurement, 9, 139-164.

Hoffman, P. J. (1959). Generating variables with arbitrary properties. Psychometrika, 24, 265-267.

Horn, J. L. (1965). A rationale and technique for estimating the number of factors in factor analysis. Psychometrika, 30, 179-185.

Humphreys, L. G., \& Ilgen, D. R. (1969). Note on a criterion for the number of common factors. Educational and Psychological Measurement, 29, 571-578.
Humphreys, L. G., \& Montanelli, R. G. (1975). An investigation of the parallel analysis criterion for determining the number of common factors. Multivariate Behavioral Research, 10, 193-205.

Linn, R. L. (1968). A monte carlo approach to the number of factors problem. Psychometrika, 33, 37-72.

Lord, F. M. (1980). Applications of item response theory to practical testing problems. Hillsdale NJ: Erlbaum.

Lord, F. M., \& Novick, M. R. (1968). Statistical theories of mental test scores. Reading MA: AddisonWesley.

McDonald, R. P. (1967). Non-linear factor analysis. Psychometric Monograph (No. 15).

McDonald, R. P. (1980). The dimensionality of tests and items. British Journal of Mathematical and Statistical Psychology, 34, 100-117.

McDonald, R. P. (1982). Linear versus nonlinear models in item response theory. Applied Psychological Measurement, 6, 379-396.

Reckase, M. D. (1979). Unifactor latent trait models applied to multifactor tests: Results and implications. Journal of Educational Statistics, 4, 207-230.

Rentz, R. R., \& Rentz, C. C. (1979). Does the Rasch model really work? Measurement in Education, 10, $1-11$.

Sympson, J. B. (1978). A model for testing with multidimensional items. In D. J. Weiss (Ed.), Proceedings of the 1977 Computerized Adaptive Testing Conference ( $\mathrm{pp}$. 82-98). Minneapolis MN: University of Minnesota.

Traub, R. E., \& Wolfe, R. G. (1981). Latent trait theories and the assessment of educational achievement. In D. C. Berliner (Ed.), Review of research in edu- 
cation-Volume 9. Washington: American Educational Research Association.

\section{Acknowledgments}

Funding for this study was provided by the American Board of Family Practice, Lexington, Kentucky. The authors thank Jamshid Etezadi for providing a copy of NOFA, a computer program for conducting nonlinear factor analyses. The authors also thank Isaac Bejar, Rod- erick McDonald, Frederic Lord, Jane Rogers, Ross Traub, and two anonymous reviewers for comments on an earlier draft of this paper. The computer program used for the residual analysis was written by Linda Murray.

\section{Author's Address}

Send requests for reprints or further information to Ronald K. Hambleton, University of Massachusetts, Hills South, Room 152, Amherst MA 01003, U.S.A. 